\title{
Besteuerung digitaler Unternehmen - die schwierige Suche nach einem europäischen Ansatz
}

\author{
Daniel Klein, Christopher Ludwig und Christoph Spengel*
}

In March 2018, the European Commission introduced two Directive proposals to target tax challenges in the era of digitalization. The first Directive proposal suggests to introduce a digital services tax of 3 percent on gross revenues from digital services as an interim measure. The second one proposes as a long-term solution to extend the permanent establishment concept to create a virtual permanent establishment if a corporation has a "significant digital presence" in a country. So far, a lack of consensus among the member states of the European Union has made successful legislation on the European level unlikely. Despite the absence of an intra-community agreement, several member states have implemented the Directive proposal of a digital services tax with various adaptions unilaterally. These actions contradict the efforts to develop agreeable tax reforms on a multilateral level. The article at hand critically evaluates the current European reform proposals.

Die digitale Transformation ist einer der größten Treiber von Veränderungen seit der industriellen Revolution. Eine Vielzahl technischer Innovationen im Bereich der Kommunikation und Informationsverarbeitung ermöglicht völlig neue Geschäftsmodelle und trägt zur kontinuierlichen Veränderung von bestehenden Modellen bei. Grundsätzlich herrscht die Ansicht, dass sich die Digitalisierung positiv auf die Gesellschaft und das Innovationsklima auswirkt. Digitale Wertschöpfungsprozesse stellen das seit über 100 Jahren existierende System der Unternehmensbesteuerung allerdings vor eine große Herausforderung. Während existierende Steuersysteme grundsätzlich an eindeutig lokalisierbare Unternehmenseinheiten und Wertschöpfungspunkte anknüpfen, scheint dies nicht mehr den global vernetzten und oft lokal ungebundenen Wertschöpfungsmodellen digitaler Unternehmen angemessen.

Auf nationaler Ebene, auf Ebene der Europäischen Union (EU) und auch in der öffentlichen Debatte wird oft davon ausgegangen, dass Digitalkonzerne die Mängel der existierenden Steuersysteme gezielt nutzen, um exzessive Steuerplanung zu betreiben. Diese Annahme wird regelmäßig durch anekdotische Beobachtungen von außergewöhnlich niedrigen Steuerbelastungen einzelner Unternehmen genährt. ${ }^{1}$ Bislang fehlt es aber weitgehend an

\footnotetext{
* Daniel Klein, Fakultät für Betriebswirtschaftslehre, Lehrstuhl für Allgemeine Betriebswirtschaftslehre und Betriebswirtschaftliche Steuerlehre II, Area Accounting \& Taxation, Universität Mannheim. Christopher Ludwig, ZEW - Leibniz-Zentrum für Europäische Wirtschaftsforschung, Mannheim.

Prof. Dr. Christoph Spengel, Fakultät für Betriebswirtschaftslehre, Lehrstuhl für Allgemeine Betriebswirtschaftslehre und Betriebswirtschaftliche Steuerlehre II, Area Accounting \& Taxation, Universität Mannheim.

Dieser Beitrag basiert auf den aktuellen Arbeiten an der Universität Mannheim und dem ZEW - Leibniz-Zentrum für Europäische Wirtschaftsforschung zum Verständnis digitaler Geschäftsmodelle und zur Entwicklung steuerlicher Reformvorschläge in Reaktion auf die zunehmende Digitalisierung der Wirtschaft. Diese Arbeiten werden im Rahmen des WissenschaftsCampus „MannheimTaxation“ und des von der Leibniz-Gemeinschaft geförderten Projekts zur Besteuerung im Zeitalter der Digitalisierung durchgeführt. Christopher Ludwig und Daniel Klein danken der Graduate School of Economic and Social Sciences der Universität Mannheim für finanzielle Förderung.

1 Bastian Brinkmann/Frederik Obermaier: Apples Steuertricks kosten EU-Staaten mindestens vier Milliarden Euro, in: Süddeutsche Zeitung, 21. Juni 2018.
} 
empirischer Evidenz, dass Konzerne mit digitalen Geschäftsmodellen ihre Steuerbelastung tatsächlich systematisch aggressiver reduzieren als klassische Unternehmen. Die vorgebrachten Kalkulationen der Europäischen Kommission hinsichtlich eines Steuergefälles zwischen traditionellen und digitalen Unternehmen haben sich als nicht tragfähig erwiesen. $^{2}$

Weniger umstritten in der Politik sowie in der Wissenschaft ist jedoch, dass das derzeitige System der internationalen Unternehmensbesteuerung nicht mehr zeitgemäß ist. Dies bestärkt bei vielen politischen Entscheidungsträgern die Befürchtung, dass Staatseinnahmen durch eine Erosion des Steuersubstrats und die übermäßige Gewinnverlagerung von multinationalen Unternehmen leiden. ${ }^{3}$ Seit Beginn des Aktionsplans der Organisation für wirtschaftliche Zusammenarbeit und Entwicklung (Organisation for Economic Co-operation and Development, $\mathrm{OECD})^{4}$ zur Bekämpfung der Gewinnverkürzung und Gewinnverlagerung (Base Erosion and Profit Shifting, BEPS) im Jahr 2013 wird kontinuierlich an Reformvorschlägen zur Anpassung des Systems der Unternehmensbesteuerung gearbeitet. In den letzten zwei Jahren haben diese Reformüberlegungen an Dynamik gewonnen. Nicht mehr nur auf Ebene der OECD wird ein umfassendes und globales Reformpaket erarbeitet, sondern auch die Europäische Kommission hat im März 2018 zwei Richtlinienvorschläge zur Besteuerung digitaler Unternehmen veröffentlicht. Der erste Richtlinienvorschlag sieht die Einführung einer umsatzbasierten Digitalsteuer in Höhe von 3 Prozent auf digitale Umsätze vor. Diese sollte als Übergangslösung eingeführt werden. Der zweite Vorschlag, der die Ausweitung des Betriebsstättenkonzepts basierend auf der Existenz einer „signifikanten digitalen Präsenz" vorsieht, sollte als längerfristige Lösung entwickelt werden. Beide Richtlinienvorschläge sind bisher nicht umgesetzt worden. Trotz Anpassungen durch das Europäische Parlament und den Europäischen Rat wurde die bei Steuerfragen notwendige Einstimmigkeit nicht erreicht. ${ }^{5}$ Obwohl es im Europäischen Parlament eine überwiegende Mehrheit für den modifizierten Vorschlag gegeben hat, ${ }^{6}$ ist im März 2019, fast ein Jahr nach dessen Veröffentlichung, die Übergangslösung einer Digitalsteuer mit den Gegenstimmen Schwedens, Finnlands, Irlands und Dänemarks im Rat der EU abgelehnt worden. Vorerst wird auf eine globale Reform des Steuersystems durch die OECD gewartet. Sollte diese nicht bis Ende 2020 umgesetzt werden, könnten die Richtlinienvorschläge der Europäischen Kommission wieder an die Spitze der politischen Agenda gesetzt werden.

Unabhängig von der Zurückhaltung auf gesamteuropäischer Ebene haben einzelne EUMitgliedstaaten bereits Digitalsteuern nach dem Vorbild der Kommissionsentwürfe umge-

2 Siehe u.a. Clemens Fuest: Digitalisierung und Steuerpolitik, in: ifo Schnelldienst 14/2018, S. $22-23$.

3 Bisher fehlt es jedoch an einer konsensfähigen Bemessung der Höhe dieses Steuerschadens. Siehe Jennifer Blouin/Leslie A. Robinson: Double Counting Accounting: How Much Profit of Multinational Enterprises Is Really in Tax Havens?, 21. Mai 2020, abrufbar unter: https://papers.ssrn.com/abstract=3491451 (letzter Zugriff: 3.7.2020).

4 Zur Koordination der grenzüberschreitenden Unternehmensbesteuerung erarbeitet die OECD im Bereich Steuern in Abstimmung mit ihren Mitgliedstaaten sowie weiteren Nationen (Inclusive Framework) und Zusammenschlüssen (bspw. G20) globale Referenzwerke und Musterabkommen sowie steuerliche Standards.

5 Siehe Marcin Szczepański: Interim digital services tax on revenues from certain digital services, Europäisches Parlament: Briefing, Dezember 2018.

$6 \mathrm{Zu}$ den Abstimmungsergebnissen siehe Schlussabstimmung in namentlicher Abstimmung im federführenden Ausschuss, in: Europäisches Parlament: Bericht über den Vorschlag für eine Richtlinie des Rates zum gemeinsamen System einiger Digitalsteuer auf Erträge aus der Erbringung bestimmter digitaler Dienstleistungen (COM(2018)0148 - C8-0137/2018 - 2018/0073(CNS)), 5. Dezember 2018, S. 39; Pola Schneemelcher: Digitalsteuer in der EU - wo stehen wir?, Jacques Delors Institute/Bertelsmann Stiftung: Policy Brief, 6. Mai 2019. 
setzt oder sind dabei, die Pläne zur Umsetzung zu konkretisieren. Unilaterale Alleingänge in der Steuergesetzgebung bergen allerdings das Risiko einer Zunahme der Komplexität des internationalen Steuersystems und einer eigentlich zu vermeidenden Doppelbesteuerung von multinationalen Konzernen. Die Aussicht auf kurzfristige Erhöhungen der Steuereinnahmen, der politische Wunsch, an den oft hohen Renditen digitaler Geschäftsmodelle zu partizipieren, und die Einschätzung, dass Digitalkonzerne bisher nicht fair besteuert werden, veranlassen politische Entscheidungsträger zu (vor-)schnellen Gesetzesänderungen. Der Diskurs in Staatengemeinschaften wird weder abgewartet noch mitgestaltet, der auf diese Weise globale Lösungen der Herausforderungen durch die zunehmende Digitalisierung für das Steuersystem hervorbringen könnte. Es bleibt jedoch abzuwarten, wie der zwischenstaatliche Steuerwettbewerb durch die unilateralen Entscheidungen einzelner Länder beeinflusst wird. Da es für betroffene Unternehmen schwierig sein dürfte, sich einer Umsatzbesteuerung zu entziehen, ohne vollends auf Umsatz im Marktstaat zu verzichten, dürfte sich der Steuerwettbewerb der Digitalsteuer ersichtlich von dem der Körperschaftsteuer unterscheiden.

Ziel des vorliegenden Beitrags sind die kritische Evaluation der europäischen Reformvorschläge und die Abwägung, inwieweit diese zur Lösung der steuerlichen Herausforderungen im Zeitalter der Digitalisierung beitragen können. Zunächst werden die Eigenschaften digitaler Geschäftsmodelle und die spezifischen Charakteristika dieser Wertschöpfungsmodelle erläutert, um eine Einordnung zu ermöglichen und die Herausforderungen, die durch das aktuelle Steuersystem erwachsen, zu erkennen. Anschließend werden die Richtlinienvorschläge zur Einführung einer Digitalsteuer und einer „signifikanten digitalen Präsenz" dargestellt, die zur Lösung der steuerlichen Herausforderungen im Zeitalter der Digitalisierung konzipiert worden sind. Der Artikel schließt mit einem Ausblick auf die aktuellen Reformbemühungen der OECD. Ebenso wie die europäischen Reformvorschläge stellen diese keine überzeugende Lösung für die aktuellen Herausforderungen der Besteuerung digitaler Geschäftsmodelle dar. Vielmehr bergen sie die Gefahr der ökonomisch nicht gewollten Doppelbesteuerung und der Erhöhung der Komplexität des internationalen Steuersystems.

\section{Aktuelle Herausforderungen bei der Besteuerung digitaler Unternehmen}

\section{Wertschöpfung in digitalen Geschäftsmodellen}

Trotz der zunehmenden Bedeutung der Digitalisierung für die Wirtschaft ${ }^{7}$ ist die Definition der digitalen Ökonomie bislang weder abschließend noch einheitlich. Vielmehr können nur die spezifischen Eigenschaften digitaler Geschäftsmodelle identifiziert werden. Die OECD hat in ihrem 2018 veröffentlichten Zwischenbericht zu den steuerlichen Herausforderungen, die durch die Digitalisierung verursacht wurden, drei prägende Charakteristika digitaler Geschäftsmodelle herausgestellt. ${ }^{8}$ Erstens ermöglicht die Digitalisierung eine globale Streuung der Wertschöpfung, ohne dass es in einzelnen Staaten einer physischen Präsenz bedarf. Zweitens beruhen die meisten digitalen Geschäftsmodelle auf imma-

7 Siehe Marcel Olbert/Christoph Spengel: International Taxation in the Digital Economy: Challenge Accepted, in: World Tax Journal 1/2017, S. 3-46, hier S. 4.

8 Organisation für wirtschaftliche Zusammenarbeit und Entwicklung: Tax Challenges Arising from Digitalisation - Interim Report 2018: Inclusive Framework on BEPS, Paris 2018. 
teriellen Wirtschaftsgütern. Drittens stehen die Verarbeitung von Daten und die Interaktion mit Nutzern im Zentrum.

Die ersten beiden Charakteristika werden durch die stetige Verbesserung von Kommunikationsmitteln und die Entwicklung von wertvoller Software, Algorithmen und technischem Wissen begünstigt. Die zentrale Rolle von Daten ist hingegen nur durch einen dadurch erwachsenden Wissensvorteil für Unternehmen zu erklären. Der Prozess der Transformation von Daten zu Wissen ist so zentral für viele Unternehmen, dass er einer detaillierteren Betrachtung bedarf. ${ }^{9}$

Daten, insbesondere „Big Data“, sind einer der zentralen Werttreiber für digitale Unternehmen. Sie bilden die Grundlage für bessere Entscheidungen und sind somit zentral für die Wertschöpfung - auch in traditionellen Unternehmen. Obwohl grundsätzlich akzeptiert wird, dass der Wertbeitrag von Daten bei der Unternehmensbesteuerung zu berücksichtigen ist, ist bisher allerdings noch nicht klar, wie der Wert von Daten bemessen werden soll. Grundsätzlich sind zwei Optionen möglich: ${ }^{10}$

- Wenn die Bestimmung des tatsächlichen Werts von Daten unmöglich erscheint, dann ist eine fundamentale Steuerreform hin zu einer Steuer auf die Umsätze im Land der Endverkäufe oder einer Wohnsitzbesteuerung der Anteilseigner unabdingbar. ${ }^{11}$

- Ein vielversprechenderer und politisch realisierbarer Vorschlag wäre, den Verarbeitungsprozess von Daten zu Wissen zu analysieren und die dabei entstehenden Gewinne entsprechend der aktuell existierenden Transferpreissystematik entlang der Wertschöpfung aufzuteilen.

Die von einigen Seiten gezogene Analogie zwischen Daten und natürlichen Ressourcen wie Rohöl kann kaum überzeugen. ${ }^{12}$ Vielmehr sind Daten der Ausgangspunkt eines sehr know-how-intensiven Prozesses der Transformation von Rohinformationen in Wissen durch die Entdeckung von Strukturen und Zusammenhängen in den Ausgangsinformationen. ${ }^{13}$ Dieser mehrstufige und iterative Prozess, der auch oft Data-Mining genannt wird, beginnt bei der Spezifikation des Ziels und des notwendigen Wissens für das Geschäftsmodell. Erst dann sollte mit der Sammlung und Aufbereitung der benötigten Daten begonnen werden. In einem weiteren Schritt können durch den Einsatz spezifischer Software und durch das Know-how von Mitarbeitern in den aufbereiteten Daten Muster gesucht und

9 Weitergehende Informationen dazu auch in Christoph Spengel/Christopher Ludwig: Profit Allocation in Digital Businesses, in: Transfer Pricing International 5/2019, S. 258-262; Christopher Ludwig/Marcel Olbert/ Christoph Spengel: Transfer Pricing for Data Businesses: How to apply the Arm's Length Principle to the Digital Economy, in: Pasquale Pistone/Dennis Weber (Hrsg.): Taxing the Digital Economy, Amsterdam 2019, S. 2747, hier S. 38ff.

10 Siehe Marcel Olbert/Christoph Spengel: Taxation in the Digital Economy - Recent Policy Developments and the Question of Value Creation, ZEW - Leibniz-Zentrum für Europäische Wirtschaftsforschung: Discussion Paper 19-010/2019, S. 17.

11 Siehe Michael P. Devereux/John Vella: Implications of Digitalization for International Corporate Tax Reform, in: Intertax 6/7/2018, S. 550-559, hier S. 550ff.; Wolfgang Schön: Ten Questions About Why and How to Tax the Digitalized Economy, in: Bulletin For International Taxation 4/5/2018, S. 278-292, hier S. 278ff.

12 Siehe Jocelyn Goldfein/Ivy Nguyen: Data is not the new oil, in: TechCrunch, 27. März 2018; Bernard Marr: Here's Why Data Is Not The New Oil, in: Forbes, 5. März 2018; Olbert/Spengel: Taxation in the Digital Economy, 2019.

13 Vgl. Gordon S. Linoff/Michael J. A. Berry: Data Mining Techniques. For Marketing, Sales, and Customer Relationship Management, Indianapolis 2011; Ian H. Witten/Eibe Frank/Mark A. Hall/Christopher J. Pal: Data Mining. Practical Machine Learning Tools and Techniques, Cambridge 2016. 
analysiert werden. Zuletzt muss geprüft werden, ob die gefundenen Zusammenhänge der Beantwortung der initial gestellten Frage dienen können.

Jederzeit kann es in diesem Prozess zu Anpassungen und Wiederholungen einzelner Schritte kommen. Er entspricht in vielen Fällen einem klassischen Forschungs- und Entwicklungsprozess. Ähnlich diesem kann auch das Data-Mining in verschiedenen Konzerneinheiten einer multinationalen Gruppe ausgeführt werden. In digitalen Geschäftsmodellen, etwa dem eines Streaming-Anbieters von Musik, kann es bspw. notwendig sein, die Vorlieben der Nutzer genau zu kennen, um den Service entsprechend anzupassen. Die Rohdaten zum Hörverhalten von Kunden sammeln die Anbieter in den Ländern, in denen die Kunden aktiv sind. Diese Daten können in einem anderen Land bei einem Datenbankanbieter gespeichert und vorverarbeitet werden. In einem weiteren Land können Mitarbeiter des Konzerns den unternehmensspezifischen Algorithmus zur Analyse der Daten entwickeln und anwenden. Abschließend können die Entscheidungsträger im Sitzstaat der Konzernmutter die gewonnenen Informationen auswerten und mithilfe der gewonnenen Erkenntnisse neue Märkte erschließen.

Eine zentrale Rolle nehmen in diesem Prozess die Mitarbeiter ein, die über das Wissen verfügen, den unternehmensspezifischen Algorithmus zur Auswertung der Daten zu entwickeln. Ebenso wie bei klassischen Forschungs- und Entwicklungsprozessen findet an dieser Stelle ein wesentlicher Teil der Wertschöpfung statt. Der Prozess des Data-Minings findet mittlerweile in vielen Unternehmen Anwendung. Die zentrale Herausforderung ist ebenso wie bei dem klassischen Forschungs- und Entwicklungsprozess die internationale Gewinnaufteilung des globalen Wertschöpfungsprozesses innerhalb eines Konzerns. Statt kurzsichtiger Reformen könnte die existierende Systematik der steuerlichen Transferpreise auch in diesen neuen Geschäftsmodellen als Anhaltspunkt für eine faire Besteuerung dienen. ${ }^{14}$ Falls Finanzverwaltungen nur einen unzureichenden Einblick in digitale Geschäftsmodelle erhalten und so die Funktions- und Risikoanalyse einzelner Geschäftseinheiten nicht sachgerecht durchgeführt werden kann, dann kann es zu einer Fehlallokation von Wertschöpfungsbeiträgen kommen. Dies können Unternehmen zur Verlagerung von Gewinnen in Niedrigsteuerländer nutzen und es kann zu einer Verringerung der Besteuerungsgrundlage in Hochsteuerländern, bspw. Deutschland oder Frankreich, kommen.

\section{Spannungsfeld der internationalen Unternehmensbesteuerung}

Das aktuelle System der Unternehmensbesteuerung knüpft grundsätzlich an den Ort der Wertschöpfung an. ${ }^{15}$ An diesem Ort muss regelmäßig eine Betriebsstätte oder eigenständige Konzerneinheit der multinationalen Unternehmensgruppe ansässig sein, um eine Besteuerung auszulösen. Die Gesamtsteuerbelastung eines Konzerns hängt daher grundsätzlich von den Sitzstaaten der einzelnen Gesellschaften ab. Der Sitz der Muttergesellschaft kann an einem anderen Ort sein als die Produktionsstätten, die Vertriebsgesellschaften oder die rechtliche Zugehörigkeit von Patenten und immateriellen Vermögensgegenständen. Im internationalen Steuerwettbewerb können Unternehmen Anreizen unterliegen, die

14 Siehe auch Spengel/Ludwig: Profit Allocation in Digital Businesses, 2019, S. 258.

15 Siehe Devereux/Vella: Implications of Digitalization, 2018, S. 557; Johannes Becker/Joachim Englisch: Ein größeres Stück vom Kuchen: Besteuerung der Gewinne von Google und Co., in: Wirtschaftsdienst 11/2017, S. 801-808, hier S. 802. 
Sitze ihrer Gesellschaften in Niedrigsteuerländer zu verlegen, um die Gesamtsteuerlast des Konzerns zu senken. Dieses Vorgehen, im Sinne der Anteilseigner eine Reduktion der Kosten anzustreben, wird als Steuerplanung bezeichnet und in gewissem Maße auch grundsätzlich von allen Unternehmen praktiziert. Ab welchem Ausmaß von aggressiver Steuerplanung gesprochen werden kann, ist hingegen nicht eindeutig. Die Grenze des Legalen wird jedoch nur bei der sog. Steuerhinterziehung übertreten, welche bei internationalen Unternehmen allerdings eine Seltenheit darstellt.

Die Charakteristika digitaler Unternehmen, insbesondere die vielfältigen grenzüberschreitenden Geschäfte und der hohe Einsatz von immateriellen Wirtschaftsgütern, deren Wert oft schwer zu bestimmen ist, ermöglichen es diesen, deutlich einfacher steuerplanerische Entscheidungen umzusetzen.

Erstens können digitale Produkte und Dienstleistungen unmittelbar in verschiedenen Ländern angeboten werden, ohne dass es einer physischen Präsenz, in Form einer Betriebsstätte, in den Marktstaaten bedarf. ${ }^{16}$ Eine Betriebsstätte ist definiert als eine feste Geschäftseinrichtung, die der Tätigkeit eines Unternehmens dient. ${ }^{17}$ Gemäß den international geltenden Regelungen dürfen Staaten die Gewinne eines Unternehmens nur besteuern, wenn die Geschäfte innerhalb eines Landes mittels einer Betriebsstätte ausgeführt werden. Reine Exporte, die bei digitalen Geschäften deutlich schneller zu realisieren sind, da in der Regel eine Website in Landessprache genügt, unterliegen im bisherigen System nicht der Ertragsbesteuerung.

Zweitens beruht ein Großteil der digitalen Geschäftsmodelle auf immateriellen Wirtschaftsgütern. Der Wert dieses technischen Wissens, der verwendeten Software oder Marke ist nur sehr selten objektiv messbar. Zudem sind immaterielle Wirtschaftsgüter im Gegensatz zu realen Produktionsstätten sehr mobil und können in andere Konzerngesellschaften verlagert werden. ${ }^{18}$ Die hohe Mobilität der geschäftsmodellbestimmenden Wirtschaftsgüter erhöht die Möglichkeiten für digitale Konzerne, komplexere Wertschöpfungsketten über mehrere Länder hinweg zu betreiben, und fördert somit die Globalisierung von Konzernen. Die Nutzung immaterieller Wirtschaftsgüter, wie bspw. Software, Patente oder Markenrechte, wird anderen Konzerneinheiten regelmäßig in Rechnung gestellt. Da es äußerst schwer ist, den Wert dieser Lizenzzahlungen objektiv zu bestimmen - es gibt nur sehr selten vergleichbare Marktpreise für die entsprechenden Transaktionen -, bietet dies Möglichkeiten für digitale Konzerne, Gewinne in Niedrigsteuerländer zu verlagern. Eine adäquate Gewinnallokation über Konzerneinheiten hinweg ist essenziell, um die Besteuerungsrechte an den Gewinnen eines multinationalen Konzerns entsprechend der Wertschöpfung auf die involvierten Länder zu verteilen. Dies ist bereits im Rahmen der existierenden Steuersysteme möglich und kann bspw. durch den Einsatz der geschäftsvorfallbezogenen Gewinnaufteilungsmethode realisiert werden. ${ }^{19}$ In vielen Fällen könnte durch besse-

16 Yarive Brauner/Pasquale Pistone: Adapting Current International Taxation to New Business Models: Two Proposals for the European Union, in: Bulletin for International Taxation 12/2020, S. 681-687, hier S. 681.

17 Vgl. $\$ 12$ Abgabenordnung.

18 Vgl. Lisa Spinosa/Vikram Chand: A Long-Term Solution for Taxing Digitalized Business Models: Should the Permanent Establishment Definition Be Modified to Resolve the Issue or Should the Focus Be on a Shared Taxing Rights Mechanism?, in: Intertax 6/7/2018, S. 476-494, hier S. 479.

19 Siehe Otto H. Jacobs/Dieter Endres/Christoph Spengel/Andreas Oestreicher/Andreas Schumacher: Internationale Unternehmensbesteuerung: Deutsche Investitionen im Ausland. Ausländische Investitionen im Inland, München 2016, S. $575 \mathrm{ff}$. 
re Analyse des betreffenden Geschäftsmodells und die Unterstützung bei Transferpreisregelungen im Rahmen der existierenden Systematik die Besteuerung von digitalen Unternehmen sachgerechter durchgeführt werden. ${ }^{20}$ Die fortschreitende Globalisierung und Unabhängigkeit digitaler Geschäftsmodelle von einer physischen Präsenz in Marktstaaten und der dadurch mangelnde steuerliche Nexus stellen wesentliche Herausforderungen für die Besteuerung im Zeitalter der Digitalisierung dar.

Zusammengefasst ergeben sich durch die Digitalisierung zwei zentrale Herausforderungen für die internationale Unternehmensbesteuerung: Erstens erfasst die aktuelle Definition von Betriebsstätten nicht die reinen Exporte digitaler Geschäfte; zweitens ermöglicht die mangelhafte Anwendung der Transferpreissystematik digitalen Unternehmen, Gewinne übermäßig in Niedrigsteuerländer zu verlagern. In Anbetracht dieser Herausforderungen und des Bedürfnisses von Marktstaaten, an den Gewinnen profitabler Digitalunternehmen zu partizipieren, wurden von der Europäischen Kommission zwei Richtlinienvorschläge entwickelt.

\section{Die Richtlinienvorschläge der Europäischen Kommission zur Besteuerung digitaler Unternehmen}

\section{Der Vorschlag zur Einführung einer Digitalsteuer}

Der erste Vorschlag der Kommission beinhaltet die Einführung einer neuen europaweiten Digitalsteuer als Übergangslösung bis zur Ausarbeitung der Details des zweiten Vorschlags, der eine Ausweitung des Betriebsstättenkonzepts bei „signifikanter digitaler Präsenz" als langfristige Lösung vorsieht (siehe hierzu den nächsten Abschnitt). ${ }^{21}$

Die Digitalsteuer soll nach dem Plan der Europäischen Kommission eine Steuer mit objektiver Bemessungsgrundlage darstellen, die im Wesentlichen Umsätze aus der Bereitstellung bestimmter digitaler Dienstleistungen besteuert. Dies sind Dienstleistungen, bei denen Nutzer einen hohen Anteil an der Wertschöpfung haben. Dies ist der Fall, wenn Nutzer des Dienstes einen wesentlichen Beitrag zu dessen Erstellung leisten. Basierend auf diesen Eigenschaften definiert die Europäische Kommission drei Dienstleistungsarten als steuerpflichtig: ${ }^{22}$

1. Erträge aus der Platzierung von Onlinewerbung;

2. Erträge aus digitalen Vermittlungsgeschäften, die es Nutzern erlauben zu interagieren, um bspw. auch Güter und Dienstleistungen direkt zu handeln;

3. Erträge aus der Veräußerung von Nutzerdaten.

Allerdings definiert die Kommission auch direkt Ausnahmen dieser Regelungen für digitale Plattformen, die hauptsächlich genutzt werden, um digitale Inhalte, Kommunikationsmittel oder Bezahldienste anzubieten. Dies bedeutet, dass Abonnementgebühren für digitale Inhalte sowie E-Commerce-Plattformen von der Steuer befreit sein sollen. Bei diesen

20 Siehe Olbert/Spengel: International Taxation in the Digital Economy, 2017, S. 33.

21 Siehe Europäische Kommission: Vorschlag für eine Richtlinie des Rates zum gemeinsamen System einer Digitalsteuer auf Erträge aus der Erbringung bestimmter digitaler Dienstleistungen, $\operatorname{COM(2018)~} 148$ final; Europäische Kommission: Vorschlag für eine Richtlinie des Rates zur Festlegung von Vorschriften für die Unternehmensbesteuerung einer signifikanten digitalen Präsenz, COM(2018) 147 final.

22 Vgl. Europäische Kommission: Fair Taxation of the Digital Economy, abrufbar unter: https://ec.europa.eu/taxa tion_customs/business/company-tax/fair-taxation-digital-economy_en (letzter Zugriff: 21.2.2020). 
Geschäftsmodellen spielen Nutzer nach Auffassung der Europäischen Kommission nur eine untergeordnete Rolle für die Wertschöpfung. ${ }^{23}$ Nach dieser Definition würden Netzwerke wie Facebook, Suchmaschinenanbieter wie Alphabet, Plattformen wie Airbnb und eBay sowie Twitter durch den Verkauf von Nutzerdaten von der Steuer getroffen. Dahingegen würden Bezahldienste wie PayPal, die Onlinestreaming-Anbieter Spotify und Netflix oder der Onlinehändler Zalando von der Steuer ausgenommen werden. ${ }^{24}$ Die bisherige Formulierung des Richtlinienvorschlags ist allerdings nicht trennscharf und bei einigen Geschäftsmodellen, insbesondere bei Mischformen, würde die Anwendbarkeit der Steuer eine Einzelfallentscheidung bleiben.

Steuersubjekte sind des Weiteren nach Maßgabe des Richtlinienvorschlags nur Unternehmen, die folgende zwei Größenkriterien erfüllen: ${ }^{25}$

1. Die Gesamtsumme der weltweiten Konzernumsätze muss 750 Millionen Euro innerhalb eines Finanzjahres überschreiten;

2. Im selben Jahr muss die Gesamtsumme der steuerbaren Umsätze in der EU, d.h. Umsätze durch die Veräußerung von digitalen Dienstleistungen, 50 Millionen Euro übersteigen.

Für die Überschreitung dieser Größengrenzen ist allerdings unerheblich, ob die Gesellschaften ihren Hauptsitz innerhalb oder außerhalb der EU haben. Es ist immer der konsolidierte Gesamtumsatz der Unternehmen zu betrachten. ${ }^{26}$ Daher können auch kleinere Konzerneinheiten in den Anwendungsbereich der Digitalsteuer fallen, wenn die Grenzen im Konzernverbund überschritten werden. ${ }^{27}$ Die Europäische Kommission begründet die Wahl der grundsätzlich arbiträren Grenze von 750 Millionen Euro - die so auch im Rahmen anderer steuerlicher Vorschriften Anwendung findet oder diskutiert wurde - wie folgt: Erstens werden durch diese Grenze kleine und mittelständische Unternehmen sowie Start-ups von zu hohen Verwaltungsaufwendungen durch die Steuer verschont. Zweitens sollen nur Unternehmen von der Steuer getroffen werden, die genügend Marktmacht haben, um von der Wertschöpfung durch Nutzer zu profitieren. Drittens geht die Kommission davon aus, dass große Unternehmen mehr Steuerplanung als kleinere Unternehmen betreiben. ${ }^{28}$ Zudem soll die Größenbegrenzung der Vereinfachung der Bestimmung des Anwendungsbereichs der neu vorgeschlagenen Steuer dienen. Die Begrenzung hinsichtlich der digitalen Umsätze innerhalb der EU soll den Anwendungsbereich der Steuer präzisie-

23 Siehe Europäische Kommission: Vorschlag für eine Richtlinie des Rates zum gemeinsamen System einer Digitalsteuer, 2018, S. 10f.

24 Vgl. Wei Cui: The Digital Services Tax: A Conceptual Defense, The Peter A. Allard School of Law: Working Paper, 26. Oktober 2018, S. 5; Gary Clyde Hufbauer/Lu Zhiyao: The European Union's Proposed Digital Services Tax: A De Facto Tariff, Peterson Institute for International Economics: Policy Brief 18-15/2018, S. 9.

25 Siehe Europäische Kommission: Vorschlag für eine Richtlinie des Rates zum gemeinsamen System einer Digitalsteuer, 2018, S. 12; Johannes Becker/Joachim Englisch: EU Digital Services Tax: A Populist and Flawed Proposal, in: Kluwer International Tax Blog, 16. März 2018, abrufbar unter: http://kluwertaxblog.com/2018/03/16 /eu-digital-services-tax-populist-flawed-proposal/ (letzter Zugriff: 9.7.2020).

26 Siehe Europäische Kommission: Vorschlag für eine Richtlinie des Rates zum gemeinsamen System einer Digitalsteuer, 2018, S. 12f.

27 Vgl. Martin Nieminen: The Scope of the Commission's Digital Tax Proposals, in: Bulletin for International Taxation 11/2018, S. 664-675, hier S. 665.

28 Siehe Europäische Kommission: Vorschlag für eine Richtlinie des Rates zum gemeinsamen System einer Digitalsteuer, 2018, S. 12; Nieminen: The Scope of the Commission's Digital Tax Proposals, 2018, S. 665. 
ren und Unternehmen, die nicht in der Digitalwirtschaft oder nicht innerhalb des europäischen Marktes aktiv sind, von der Steuer verschonen. ${ }^{29}$

Die arbiträr gewählten Größengrenzen können allerdings zu realen ökonomischen Verzerrungen führen. Die Regelungen stehen unter Verdacht, dass sie gezielt US-amerikanische Unternehmen treffen sollen und europäische Unternehmen von dieser Sondersteuer ausnehmen. ${ }^{30}$ Die Europäische Kommission bestreitet dieses Problem in ihrer Folgenabschätzung zur Einführung der Digitalsteuer, erkennt allerdings an, dass höhere Grenzen als die im Richtlinienvorschlag diesen Effekt haben könnten. ${ }^{31}$ Negative Auswirkungen auf den fairen Wettbewerb ergeben sich allerdings bei jeder Umsatzgrenze, da Unternehmen mit Umsätzen knapp oberhalb der Schwellen gegenüber vergleichbaren Unternehmen, die diese Grenzen soeben nicht reißen, diskriminiert werden. ${ }^{32}$ Zudem kreieren starre Größengrenzen, ab denen mit deutlich höheren Kosten durch zusätzliche Steuerzahlungen zu rechnen ist, Fehlanreize für Unternehmen, ihr Wachstum zu begrenzen. ${ }^{33}$

Gemäß dem Richtlinienvorschlag sollen die betroffenen digitalen Umsätze mit einer Steuer in Höhe von 3 Prozent proportional besteuert werden. Als steuerbare Umsätze zählen alle digitalen Bruttoumsätze, abzüglich Umsatzsteuern und vergleichbarer Verbrauchsteuern. Eine Bruttoumsatzbesteuerung weicht allerdings fundamental von der bisherigen Systematik der Nettogewinnbesteuerung ab. Dadurch können sich Wettbewerbsnachteile für betroffene Unternehmen ergeben. ${ }^{34}$ Die effektive Steuerlast eines Unternehmens ist bei einer Bruttoumsatzsteuer negativ mit der Rentabilität eines Unternehmens assoziiert. Bei einem beispielhaften Umsatz in Höhe von 100 Geldeinheiten und einer Rentabilität von 5 Prozent würde die Steuer in Höhe von 3 Geldeinheiten einer Gewinnbesteuerung von 60 Prozent entsprechen. Bei einer Rentabilität von 20 Prozent und gleicher Umsatzhöhe würde die Bruttoumsatzbesteuerung einer Gewinnbesteuerung von nur 15 Prozent entsprechen. Selbst wenn die Steuer, wie vorgesehen, von der Körperschaftsteuer abzugsfähig sein sollte, kann die Höhe der Bruttoumsatzbesteuerung die Körperschaftsteuer deutlich übersteigen. Damit leiden Unternehmen mit geringer Rentabilität übermäßig unter der neuen

29 Siehe Europäische Kommission: Commission Staff Working Document. Impact Assessment. Accompanying the document. Proposal for a Council Directive laying down rules relating to the corporate taxation of a significant digital presence and Proposal for a Council Directive on the common system of a digital services tax on revenues resulting from the provision of certain digital services, $\operatorname{SWD}(2018) 81$ final/2, S. 67f.; Nieminen: The Scope of the Commission's Digital Tax Proposals, 2018, S. 666.

30 Siehe Clemens Fuest/Volker Meier/Florian Neumeier/Daniel Stöhlker: Die Besteuerung der Digitalwirtschaft. $\mathrm{Zu}$ den ökonomischen und fiskalischen Auswirkungen der EU-Digitalsteuer, München 2018, S. 28; Hufbauer/ Zhiyao: The European Union's Proposed Digital Services Tax, 2018, S. 5; Nieminen: The Scope of the Commission's Digital Tax Proposals, 2018, S. 665; Becker/Englisch: EU Digital Services Tax, 2018.

31 Siehe Europäische Kommission: Impact Assessment, 2018, S. 69.

32 Vgl. Fuest/Meier/Neumeier/Stöhlker: Die Besteuerung der Digitalwirtschaft, 2018, S. 17; Helge Sigurd NæssSchmidt/Martin H. Thelle/Bruno Basalisco/Palle Sørensen/Bjarke Modvig Lumby: The Proposed EU Digital Services Tax: Effects on welfare, growth and revenues, Copenhagen Economics, September 2018, S. 13.

33 Vgl. Fuest/Meier/Neumeier/Stöhlker: Die Besteuerung der Digitalwirtschaft, 2018, S. 17; Næss-Schmidt/Thelle/Basalisco/Sørensen/Lumby: The Proposed EU Digital Services Tax, 2018, S. 13; PricewaterhouseCoopers: European Commission Proposals for Directives regarding fair taxation of the digital economy ("Digital Tax Package"), 16. Mai 2018, S. 9; Nieminen: The Scope of the Commission's Digital Tax Proposals, 2018, S. 666.

34 Siehe Fuest/Meier/Neumeier/Stöhlker: Die Besteuerung der Digitalwirtschaft, 2018, S. 18; Matthias Bauer: Five Questions about the Digital Services Tax to Pierre Moscovici, European Centre for International Political Economy: ECIPE Occasional Paper 4/2018, S. 2, 9; Krister Andersson: Should We Use Value Creation or Destination as a Basis for Taxing Digital Businesses? - Krister Andersson's Comments on the 2018 Klaus Vogel Lecture Given by Professor Michael Devereux, in: Bulletin for International Taxation 12/2018, S. 684-689, hier S. 687. 
Digitalsteuer. ${ }^{35}$ Grundsätzlich führt eine Bruttoumsatzbesteuerung zu großen Verzerrungen, da die mit den Einnahmen in Verbindung stehenden Aufwendungen nicht berücksichtigt werden. Im Verlustfall, der auch durch einen Expansionskurs von Unternehmen auftreten kann, führt die Bruttoumsatzbesteuerung zu einer starken Verschärfung der finanziellen Belastung von Unternehmen und eine Substanzbesteuerung kann nicht ausgeschlossen werden.

Die Einnahmen der Digitalsteuer sollen anhand eines Schlüssels an die EU-Mitgliedstaaten verteilt werden, in denen Umsätze generiert werden. Dieser Schlüssel unterscheidet sich je nach Umsatzkategorie. Im Falle von Umsätzen, die durch die Darstellung von Werbung auf digitalen Endgeräten erzielt werden, soll die Schlüsselung anhand der Anzahl der Einblendungen der Werbung auf Endgeräten von Nutzern an die beteiligten Mitgliedstaaten erfolgen. ${ }^{36}$ Steuereinnahmen, die durch Umsätze aus dem Verkauf von Nutzerdaten erzielt werden, sollen basierend auf der Anzahl der Nutzer, deren Daten veräußert werden, an die Länder der Wohnsitze der Nutzer allokiert werden. Die Einkünfte aus Steuereinnahmen durch die Bereitstellung von Interaktionsplattformen sollen entsprechend den Angaben in Nutzerprofilen oder dem Ort der durchgeführten Transaktion auf die beteiligten Länder verteilt werden. ${ }^{37}$ Der Ort, an dem Nutzer aktiv werden, soll über die Internetprotokoll-Adresse (IP-Adresse) festgestellt werden. Es ist jedoch noch fraglich, ob dieses Netzwerkprotokoll als Grundlage für eine rechtssichere Aufteilung von Steuereinnahmen dienen kann. ${ }^{38}$ Um den administrativen Aufwand für Unternehmen zu begrenzen, soll - ähnlich wie bei der Umsatzsteuer schon angewendet - mittels einer One-Stop-Shop-Lösung die Registrierung nur in einem Land der EU notwendig sein. ${ }^{39}$

\section{Das Konzept der "signifikanten digitalen Präsenz": die virtuelle Betriebsstätte}

Der zweite Richtlinienvorschlag der Europäischen Kommission strebt eine langfristige Lösung zur Besteuerung digitaler Unternehmen an. Er sieht einen neuen steuerlichen Anknüpfungspunkt für Unternehmen ohne physische Präsenz, aber mit wesentlichen („signifikanten“) digitalen Aktivitäten in EU-Mitgliedstaaten vor. ${ }^{40}$ Die Mitgliedstaaten sollen die Möglichkeit haben, die Gewinne von Unternehmen innerhalb ihres Hoheitsgebietes zu besteuern, obwohl die Unternehmen nach bisheriger Definition keine Betriebsstätte in diesem Land unterhalten. ${ }^{41}$ Das Konzept der „signifikanten digitalen Präsenz“ soll demnach die Definition von Betriebsstätten auch auf digitale Betriebsstätten ausweiten. ${ }^{42}$ Im Grund-

35 Bauer: Five Questions, 2018, S. 9.

36 Siehe Europäische Kommission: Vorschlag für eine Richtlinie des Rates zum gemeinsamen System einer Digitalsteuer, 2018, S. 13f.; Fuest/Meier/Neumeier/Stöhlker: Die Besteuerung der Digitalwirtschaft, 2018, S. 10.

37 Siehe Europäische Kommission: Vorschlag für eine Richtlinie des Rates zum gemeinsamen System einer Digitalsteuer, 2018, S. 13f.

38 Vgl. Nieminen: The Scope of the Commission's Digital Tax Proposals, 2018, S. 672f.

39 Siehe Europäische Kommission: Vorschlag für eine Richtlinie des Rates zum gemeinsamen System einer Digitalsteuer, 2018, S. 15.

40 Siehe Europäische Kommission: Vorschlag für eine Richtlinie des Rates zur Festlegung von Vorschriften für die Unternehmensbesteuerung einer signifikanten digitalen Präsenz, 2018, S. 3.

41 Ebenda, S. 4f.

42 Vgl. Nieminen: The Scope of the Commission's Digital Tax Proposals, 2018, S. 664; Europäische Kommission: Vorschlag für eine Richtlinie des Rates zur Festlegung von Vorschriften für die Unternehmensbesteuerung einer signifikanten digitalen Präsenz, 2018, S. 8f. 
satz ist diese Ausweitung somit kompatibel mit der bisherigen Systematik der internationalen Unternehmensbesteuerung. ${ }^{43}$

Eine virtuelle Betriebsstätte soll gemäß dem Vorschlag der Europäischen Kommission in einem Mitgliedstaat gegeben sein, wenn ein Unternehmen in diesem Land signifikante digitale Aktivitäten ausübt. Diese sind gegeben, wenn ein Unternehmen digitale Dienstleistungen über digitale Medien anbietet und eine oder mehrere der folgenden Grenzen innerhalb eines steuerlichen Veranlagungszeitraums durch das Unternehmen selbst - oder im Verbund mit anderen Konzernunternehmen - überschritten werden: ${ }^{44}$

1. Die Summe der Umsätze aus der Erbringung digitaler Dienstleistungen übersteigt 7 Millionen Euro;

2. Die Anzahl der Nutzer digitaler Dienstleistungen übersteigt 100.000;

3. Die Anzahl der geschlossenen Geschäftsverträge übersteigt 3.000.

Diese Kriterien sind laut der Europäischen Kommission geeignet, um den digitalen Fußabdruck von Unternehmen zu approximieren und um verschiedene digitale Geschäftsmodelle zu erfassen. Zudem sollen diese Grenzwerte eine faire und vergleichbare Behandlung von Unternehmen in verschiedenen Mitgliedstaaten gewährleisten und den Anwendungsbereich nur auf große Unternehmen beschränken, um keine zusätzlichen Verwaltungskosten für kleine Unternehmen entstehen zu lassen. ${ }^{45}$

Die gewählten Grenzwerte führen allerdings zu zwei wesentlichen Problemen. Erstens sind sie inkonsistent und können das Entstehen einer digitalen Betriebsstätte zu sehr unterschiedlichen und vermutlich bereits sehr geringen Grenzwerten bedingen. So führt die Überschreitung von 100.000 Nutzern bereits zur Begründung einer digitalen Betriebsstätte. Wird angenommen, dass für je 1,50 Euro eine mobile Applikation an jeden dieser Nutzer verkauft wird, würde bereits bei einem Umsatz in Höhe von 1,5 Millionen Euro eine digitale Betriebsstätte entstehen. ${ }^{46}$ Dieser Umsatz ist deutlich geringer als der vorgeschlagene Grenzwert von 7 Millionen Euro. Ebenso wird durch die Vernachlässigung des Wertes von Geschäftsverträgen ein einzelner, sehr hoch dotierter Rahmenvertrag deutlich anders behandelt als eine Vielzahl kleiner Verträge mit in Summe deutlich geringerer Umsatzhöhe als der exemplarische Rahmenvertrag. ${ }^{47}$ Zweitens sind die durch diesen Richtlinienvorschlag eingeführten Kriterien deutlich schneller zu erreichen als die bisherigen Voraussetzungen für das Vorliegen einer Betriebsstätte. Finanzverwaltungen könnten, um einfacher an der Besteuerung von Unternehmensgewinnen zu partizipieren, die Anwendung der Regelungen zur „signifikanten digitalen Präsenz“ den existierenden Voraussetzungen auch bei nicht originär digitalen Unternehmen vorziehen. Dies könnte das Risiko der internationalen Doppelbesteuerung deutlich erhöhen. ${ }^{48}$

Der Anwendungsbereich der vorgeschlagenen digitalen Betriebsstätte ist deutlich breiter als der der Digitalsteuer, da lediglich der bloße Verkauf von Gütern und Dienstleistungen

43 Vgl. Fuest/Meier/Neumeier/Stöhlker: Die Besteuerung der Digitalwirtschaft, 2018, S. 23.

44 Siehe Europäische Kommission: Vorschlag für eine Richtlinie des Rates zur Festlegung von Vorschriften für die Unternehmensbesteuerung einer signifikanten digitalen Präsenz, 2018, S. 9, $18 \mathrm{f}$.

45 Ebenda, S. 9.

46 Vgl. Nieminen: The Scope of the Commission's Digital Tax Proposals, 2018, S. 675; PricewaterhouseCoopers: European Commission Proposals, 2018, S. 17.

47 Siehe Olbert/Spengel: Taxation in the Digital Economy, 2019, S. 13.

48 Vgl. PricewaterhouseCoopers: European Commission Proposals, 2018, S. 17. 
über Online-Netzwerke und Kommunikationsdienstleistungen ausgenommen sind. ${ }^{49} \mathrm{Nach}$ dem aktuellen Entwurf würden auch Online-Bezahldienste und Streaming-Anbieter die Kriterien einer digitalen Betriebsstätte erfüllen. ${ }^{50}$ In der bisherigen Steuersystematik ist die Begründung einer Betriebsstätte an wesentliche wirtschaftliche Aktivitäten geknüpft, die auch der Wertschöpfung im Unternehmen dienen können. ${ }^{51}$ Hilfstätigkeiten sind regelmäßig von der Definition der Betriebsstätte ausgenommen. Durch diesen Richtlinienvorschlag würde allerdings auch die reine Nutzung von digitalen Dienstleistungen eine Betriebsstätte begründen können. Eine Ausweitung der Betriebsstättenklassifikation auf reine Exportsachverhalte ist höchst kritisch zu sehen, da dies die Gefahr der internationalen Doppelbesteuerung deutlich erhöht, wenn alle Länder ihren Zugriff auf die Unternehmensgewinne ausweiten möchten. Insbesondere im Verhältnis zu Drittstaaten kann es hier durch gegebenenfalls mangelnde Anpassungen in internationalen Abkommen schnell zu einer vermehrten Doppelbesteuerung kommen.

Auch nach dem Kommissionsvorschlag bleibt nach Begründung einer Betriebsstätte die - wie in der aktuellen Besteuerungssystematik - komplexe internationale Zuteilung von Gewinnen. Der Richtlinienvorschlag löst diese Transferpreisproblematiken nicht auf. Vielmehr bleibt es bei der Notwendigkeit, die Geschäftsmodelle der digitalen Unternehmen genau zu analysieren und eine adäquate grenzüberschreitende Gewinnaufteilung vorzunehmen.

\section{Ökonomische Auswirkungen}

Die ökonomischen Folgen der Umsetzung dieser beiden Richtlinienvorschläge sind schwer abzuschätzen. Eine erste Folgenabschätzung der Europäischen Kommission, die mit den Vorschlägen veröffentlicht wurde, geht von zusätzlichen Steuereinnahmen in Höhe von jährlich knapp 5 Milliarden Euro aus. ${ }^{52}$ In Anbetracht der erhöhten Doppelbesteuerungsrisiken und der hohen Komplexität, die durch die neuen Maßnahmen bedingt wären, scheinen die europaweiten Mehreinnahmen nicht überzeugend. Die Umverteilung der Steuereinnahmen auf die EU-Mitgliedstaaten würde einzelnen Ländern voraussichtlich nur sehr marginale Mehreinnahmen bescheren. In einer anderen Studie werden deutlich niedrigere Gesamteinnahmen durch die Digitalsteuer geschätzt. Fuest et al. zeigen in ihrer Analyse, dass nur mit Mehreinnahmen in Höhe von etwa 3,9 Milliarden Euro gerechnet werden kann. ${ }^{53}$ Dies entspricht in etwa 1,2 Prozent der Einnahmen aus der Körperschaftsteuer in der EU. ${ }^{54}$ Diese Einnahmen sind in den meisten Ländern grundsätzlich vernachlässigbar. ${ }^{55}$

49 Siehe Europäische Kommission: Impact Assessment, 2018, S. 44; Europäische Kommission: Vorschlag für eine Richtlinie des Rates zur Festlegung von Vorschriften für die Unternehmensbesteuerung einer signifikanten digitalen Präsenz, 2018, S. 8f.

50 Vgl. Nieminen: The Scope of the Commission's Digital Tax Proposals, 2018, S. 665; Fuest/Meier/Neumeier/ Stöhlker: Die Besteuerung der Digitalwirtschaft, 2018, S. 33.

51 Vgl. Becker/Englisch: Ein größeres Stück vom Kuchen, 2017, S. 804.

52 Siehe Europäische Kommission: Impact Assessment, 2018, S. 70f.

53 Vgl. Fuest/Meier/Neumeier/Stöhlker: Die Besteuerung der Digitalwirtschaft, 2018, S. 23.

54 Ebenda.

55 Die jährlichen Einnahmen in den Ländern, die diese Maßnahmen unilateral einführen, werden überwiegend auf ca. 150 Millionen bis 600 Millionen Euro geschätzt. Lediglich die spanische Regierung erwartet ein deutlich höheres Steueraufkommen, dessen Realisation allerdings höchst unsicher ist. Siehe Næss-Schmidt/Thelle/ Basalisco/Sørensen/Lumby: The Proposed EU Digital Services Tax, 2018, S. 20. 
Klein et al. haben mittels einer Kapitalmarktanalyse die Auswirkungen der Veröffentlichung der Richtlinienvorschläge auf Unternehmenswerte untersucht. Die Autoren zeigen, dass der Aktienwert der 222 vermutlich von der Digitalsteuer betroffenen Unternehmen im Zeitraum der Veröffentlichung der Richtlinienvorschläge um knapp 0,7 Prozentpunkte relativ zur Marktentwicklung gesunken ist. ${ }^{56}$ Die Aktionäre scheinen die Einführung der Digitalsteuer als wahrscheinlich wahrzunehmen und bescheinigen dieser einen negativen Effekt auf die Profitabilität der betroffenen Unternehmen. Wenn man die prozentuale Preisbewegung in absolute Veränderungen der Marktkapitalisierung übersetzt, wird sichtbar, dass im Zeitraum der Veröffentlichung der Kommissionsvorschläge die Unternehmenswerte betroffener Firmen um mindestens 52 Milliarden Euro zusätzlich zu der regulären Abwärtsbewegung des Marktes reduziert wurden. ${ }^{57}$ Zudem zeigen die Autoren, dass die Reaktion der Investoren bei Unternehmen, die mehr Steuerplanung betreiben, stärker ist. Der zusätzlichen Bruttoumsatzbesteuerung kann aus Sicht der Investoren nicht mehr ausgewichen werden.

Eine zusätzliche Besteuerung von innovativen Unternehmen kann nicht im Sinne der EU sein. Eines ihrer primären Ziele ist die Förderung von Innovationen und somit ökonomischem Wachstum. ${ }^{58}$ Daher sollten Instrumente zur Förderung dieses Ziels entwickelt werden und die Störung dieser Innovationsbranchen - durch zusätzliche Besteuerungsinstrumente - vermieden werden.

\section{Nationale Alleingänge bei der Besteuerung digitaler Unternehmen}

Die Richtlinienvorschläge der Europäischen Kommission traten aufgrund von Widerständen im Rat bisher nicht in Kraft. Bis Ende 2020 wird mit weiteren Diskussionen und dem legislativen Prozess auf eine einheitliche globale Lösung auf Ebene der OECD gewartet. Eine international einheitliche und koordinierte Abstimmung bei der Reformierung der Besteuerungssysteme wäre aufgrund der hohen Verflechtung von multinationalen Unternehmen zu begrüßen. Unter Einbezug von Experten und Praktikern könnten diese Reformen in der Theorie so ausgestaltet werden, dass sie eine willkürliche Besteuerung vermeiden und bei stetiger Veränderung flexibel bleiben. Jedoch zeigen gerade die aktuellen Diskussionen auf Ebene der OECD, zu welchen Herausforderungen und Komplexitäten verschiedene nationale Interessen bei der Findung einer solchen Lösung führen. Während exportstarke Staaten die Besteuerung am Ort des Sitzlandes belassen würden, präferieren importierende Staaten eine Lösung mit größtmöglicher Besteuerung im Marktstaat. Sollte eine Lösung auf globaler Ebene allerdings bis Ende 2020 scheitern, werden die Vorschläge der Europäischen Kommission erneut in Betracht gezogen. Einzelne EU-Mitgliedstaaten wollen allerdings nicht auf eine globale Lösung warten und haben bereits eine unilaterale Digitalsteuer eingeführt oder sind im Begriff, diese umzusetzen. Tabelle 1 stellt die entsprechenden Entwicklungen dar und zeigt die Charakteristika der jeweiligen Digitalsteuern.

56 Vgl. Daniel Klein/Christopher Ludwig/Christoph Spengel: Ring-fencing Digital Corporations: Investor Reaction to the European Commission's Digital Tax Proposals, ZEW - Leibniz-Zentrum für Europäische Wirtschaftsforschung: Discussion Paper 19-050/2019, S. 13.

57 Vgl. ebenda, S. 17.

58 Siehe Europäische Kommission: Mitteilung der Kommission. EUROPA 2020. Eine Strategie für intelligentes, nachhaltiges und integratives Wachstum, $\operatorname{KOM}(2010) 2020$ endgültig, S. 5 f. 
Alle Reformen haben gemein, dass sie die Vorschläge der Europäischen Kommission als Grundlage nutzen.

Frankreich hat im Juli 2019 die Digitalsteuer rückwirkend zum 1. Januar 2019 in Kraft treten lassen. Die zusätzliche Steuer in Höhe von 3 Prozent auf Umsätze aus digitalen Geschäften wurde allerdings aufgrund des Drucks der USA und deren Androhung, zusätzliche Zölle auf französische Produkte zu erheben, zunächst bis Ende 2020 ausgesetzt. ${ }^{59}$ Alle anderen Länder, die die Einführung einer Digitalsteuer planen (siehe Tabelle 1), halten bisher trotz des internationalen Drucks an ihren Plänen fest. Auch das Nicht-EU-Mitglied Großbritannien setzte zum 1. April 2020 eine Digitalsteuer in Kraft. Die Steuerreform weicht allerdings in einigen Punkten von den Richtlinienvorschlägen der Europäischen Kommission ab: Der Steuersatz liegt unter dem von der Europäischen Kommission vorgeschlagenen Prozentsatz, allerdings ist der Anwendungsbereich der Regelung deutlich größer, da der Grenzumsatz bereits bei 500 Millionen Euro liegt. Zudem sind Umsätze aus dem Verkauf von Daten von der Besteuerung ausgenommen. Spanien hingegen hat sich bei der im Januar 2019 unilateral verabschiedeten Digitalsteuer weitgehend an dem Richtlinienvorschlag der Europäischen Kommission orientiert. Lediglich der Grenzumsatz aus digitalen Dienstleistungen in Höhe von 3 Millionen Euro weicht von den Entwürfen der Europäischen Kommission ab.

In Österreich trat zum 1. Januar 2020 eine Digitalsteuer mit dem bislang höchsten ratifizierten Steuersatz - 5 Prozent - in Kraft. Der Anwendungsbereich ist allerdings deutlich eingeschränkter, da nur Umsätze aus Onlinewerbung der neu eingeführten Steuer unterliegen. Der Grenzumsatz für inländische digitale Umsätze ist im Vergleich zu der unilateralen Maßnahme in Spanien deutlich höher. In Österreich unterliegen Konzerne erst der Digitalsteuer, wenn die inländischen Umsätze aus Online-Marketing 25 Millionen Euro übersteigen.

Die bisher in einzelnen EU-Mitgliedstaaten und im Vereinigten Königreich eingeführten und diskutierten unilateralen Maßnahmen zur Besteuerung digitaler Dienstleistungen sind durch eine große Heterogenität geprägt. Die unterschiedliche Besteuerung digitaler Umsätze innerhalb der EU führt zu einer erhöhten Komplexität des innergemeinschaftlichen Besteuerungssystems. Diese Komplexität - ebenso wie die zusätzliche Belastung durch Bruttoumsatzsteuern - erhöht das steuerliche Risiko für digitale und innovative Unternehmen und senkt dadurch auch die Investitionsanreize für diese Unternehmen. Ebenso wie die langjährigen europaweiten Bemühungen zur Einführung einer gemeinsamen Körperschaftsteuerbemessungsgrundlage kann auch bei der Entwicklung von Lösungen für die drängenden Herausforderungen der Besteuerung im Zeitalter der Digitalisierung nur eine koordinierte Vorgehensweise innerhalb der EU befürwortet werden.

59 Siehe Süddeutsche Zeitung: Macron und Trump wollen Einigung bei Digitalsteuer, 21. Januar 2020.

integration $-3 / 2020$ 
Tabelle 1: Digitalsteuern in Europa - relevante Daten und Charakteristika

\begin{tabular}{|c|c|c|c|c|c|c|c|c|c|c|}
\hline \multirow[t]{2}{*}{ Land } & \multicolumn{4}{|c|}{ Relevante Daten } & \multicolumn{3}{|c|}{$\begin{array}{c}\text { Eigenschaften der } \\
\text { Steuer }\end{array}$} & \multicolumn{3}{|c|}{$\begin{array}{l}\text { Umsatzkatego- } \\
\text { rien }\end{array}$} \\
\hline & 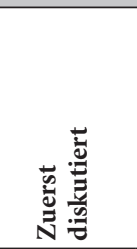 & 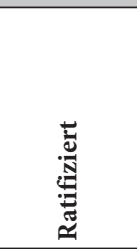 & 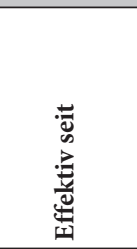 & 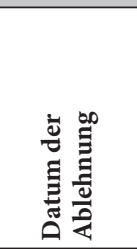 & 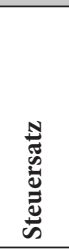 & 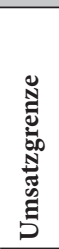 & 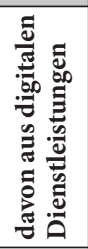 & Oే & 氖 & 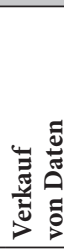 \\
\hline EU & 21.03 .2018 & - & - & 12.03 .2019 & $3 \%$ & 750 & 50 & $\mathrm{x}$ & $\mathrm{x}$ & $\mathrm{x}$ \\
\hline $\begin{array}{l}\text { Vereinigtes } \\
\text { Königreich }\end{array}$ & 29.10 .2018 & 12.03 .2019 & 01.04 .2020 & - & $2 \%$ & 550 & 28 & $\mathrm{x}$ & $\mathrm{x}$ & $\mathrm{x}$ \\
\hline Frankreich & 17.12 .2018 & 24.07.2019 & 01.01 .2019 & - & $3 \%$ & 750 & 25 & $\mathrm{x}$ & $\mathrm{x}$ & $\mathrm{x}$ \\
\hline Spanien & 23.10 .2018 & 18.01.2019 & - & - & $3 \%$ & 750 & 3 & $\mathrm{x}$ & $\mathrm{x}$ & $\mathrm{x}$ \\
\hline Italien & 12.2017 & 01.01 .2019 & 01.01 .2020 & - & $3 \%$ & 750 & 5,5 & $\mathrm{x}$ & $\mathrm{x}$ & $\mathrm{x}$ \\
\hline Österreich & 29.12 .2018 & 04.04 .2019 & 01.01 .2020 & - & $5 \%$ & 750 & 25 & $\mathrm{x}$ & & \\
\hline $\begin{array}{l}\text { Tschechische } \\
\text { Republik }\end{array}$ & 30.04 .2019 & - & 01.01 .2021 & - & $5 \%$ & 750 & 3,7 & $\mathrm{x}$ & $\mathrm{x}$ & $\mathrm{x}$ \\
\hline Belgien & 17.01.2019 & - & - & - & $3 \%$ & 750 & 5 & & & $\mathrm{x}$ \\
\hline Polen & 29.04 .2019 & - & 01.07 .2020 & - & $1,5 \%$ & - & - & $\mathrm{x}$ & $\mathrm{x}$ & $\mathrm{x}$ \\
\hline
\end{tabular}

Quelle: Klein/Ludwig/Spengel: Ring-fencing Digital Corporations, 2019; Accountancy Europe: Taxing the Digital Economy. How to capture the value created by tech companies. Factsheet, Brüssel 2019; Grant Thornton: Digital services tax in Europe, 25. Februar 2019, abrufbar unter: https://www.granttho rnton.global/en/insights/articles/digital-services-tax-in-europe/ (letzter Zugriff: 9.7.2020); Elke Asen: What European OECD Countries Are Doing about Digital Services Taxes, in: Tax Foundation, 22. Juni 2020.

\section{Globale Reformen der OECD stehen den EU-Reformvorschlägen voran}

Die weitere Diskussion der Richtlinienvorschläge wurde mangels Konsens zwischen den EU-Mitgliedstaaten im März 2019 vorerst eingestellt. Zudem wird auf Ebene der OECD derzeit über eine tiefgreifende Reform der weltweiten Besteuerung von Unternehmen diskutiert, die auf zwei Säulen basiert. ${ }^{60}$

Die erste Säule sieht einen sog. Unified Approach vor, mit dem Ziel, die Besteuerungsrechte zwischen Ländern neu zu verteilen. Auf diese Weise sollen die Marktstaaten, in de-

60 Die Vorschläge der OECD finden sich unter: Organisation für wirtschaftliche Zusammenarbeit und Entwicklung: Secretariat Proposal for a "Unified Approach" under Pillar One. Public consultation document, 9 October 2019 - 12 November 2019, abrufbar unter: https://www.oecd.org/tax/beps/public-consultation-documentsecretariat-proposal-unified-approach-pillar-one.pdf (letzter Zugriff: 9.7.2020); und Organisation für wirtschaftliche Zusammenarbeit und Entwicklung: Global Anti-Base Erosion Proposal ("GloBE") - Pillar Two. Public consultation document, 8 November 2019 - 2 December 2019, abrufbar unter: https://www.oecd.org/ta $\mathrm{x} /$ beps/public-consultation-document-global-anti-base-erosion-proposal-pillar-two.pdf.pdf (letzter Zugriff: 9.7.2020).

$\mathrm{Zu}$ weiteren Einschätzungen der aktuellen Reformvorschläge der OECD siehe Leonie Fischer et al.: Die OECD-Vorschläge für eine weltweite Reform der Unternehmensbesteuerung - eine Wende zum Schlechten?, ZEW - Leibniz-Zentrum für Europäische Wirtschaftsforschung: ZEW policy brief 1/2020. 
nen vor allem die Nutzer von Produkten und Leistungen sitzen, stärker als bisher am Steueraufkommen beteiligt werden. Dafür sollen die weltweiten, konsolidierten Unternehmensgewinne in Routine- und Residualgewinne aufgeteilt werden. Routinegewinne entsprechen den unternehmensspezifischen Kapitalkosten. Dahinter verbirgt sich die Idee einer „normalen“ marktmäßigen Verzinsung des eingesetzten Kapitals. Residualgewinne, auch Übergewinne genannt, entsprechen der Differenz zwischen konsolidierten Unternehmensgewinnen und den Routinegewinnen. Routinegewinne sollen entsprechend dem geltenden Verrechnungspreissystem auf die einzelnen Länder verteilt werden („Amount B“). Ein Teil des Residualgewinns wird, proportional zur Höhe der entsprechenden Umsätze, auf alle Länder verteilt, in denen ein Unternehmen Einnahmen erzielt („Amount A“). Der verbleibende Rest des Residualgewinns wird nach der bestehenden Transferpreissystematik aufgeteilt („Amount C“).

Die zweite Säule zielt auf die globale Bekämpfung der Gewinnreduzierung durch Aushöhlung der Besteuerungsgrundlagen ab (Global Anti-Base Erosion, GloBE). Den verbleibenden Risiken einer Gewinnverlagerung durch multinationale Unternehmen soll entgegengewirkt werden, indem eine koordinierte globale Mindeststeuer und ein weitreichendes Abzugsverbot für grenzüberschreitende Transaktionen eingeführt werden. Dieser Vorschlag sieht nicht - im Gegensatz zu der innerhalb der EU diskutierten gemeinsamen konsolidierten Körperschaftsteuerbemessungsgrundlage - die Definition einer gemeinsamen steuerlichen Bemessungsgrundlage und die Aufteilung dieser anhand spezifischer Faktoren vor.

Die Reformvorschläge der OECD sind potenziell zu weitgehend. Marktstaaten Besteuerungsrechte auf einen willkürlich gewählten Anteil an Gewinnen einzuräumen - auch ohne eine physische Präsenz in den entsprechenden Ländern -, ist überzogen und erhöht sowohl die Steuerkomplexität als auch den Verwaltungsaufwand. Zudem sind durch die Umsetzung der europäischen Anti-Steuervermeidungsrichtlinie (Anti-Tax Avoidance Directive, ATAD) vom 12. Juli 2016 in allen Mitgliedstaaten wesentliche Mindeststandards zur Gewinnverlagerung multinationaler Konzerne eingeführt oder verschärft worden. ${ }^{61}$ Die Regelungen zur Hinzurechnungsbesteuerung sichern bereits weitgehend das Besteuerungsrecht von Sitzländern. In vielen Ländern bestehen zudem schon jetzt wirkungsvolle Abzugsbeschränkungen bspw. für die steuerliche Absetzbarkeit von Zins- und Lizenzgebühren. Auch die umfassende amerikanische Steuerreform 2018 beinhaltet Elemente, die eine Mindestbesteuerung bewirken. Die von der OECD ins Auge gefasste Ausweitung der Besteuerung erhöht das Risiko einer Doppelbesteuerung erheblich.

Die dynamisch voranschreitende Digitalisierung bildet die Grundlage für vollkommen neue Geschäftsmodelle und es bedarf einer wettbewerbsgerechten Besteuerung der damit einhergehenden Wertschöpfung. Die - im Verhältnis zu den Reformvorschlägen der OECD enger gefassten - Richtlinienvorschläge der Europäischen Kommission adressieren diese wesentliche Herausforderung für das Steuersystem im 21. Jahrhundert. Dennoch können die Entwürfe in ihrer jetzigen Fassung nicht überzeugen. Die als Übergangslösung vorgesehene Bruttoumsatzbesteuerung einer spezifischen Industrie ist in ihrer vorgeschla-

61 Richtlinie (EU) 2016/1164 des Rates vom 12. Juli 2016 mit Vorschriften zur Bekämpfung von Steuervermeidungspraktiken mit unmittelbaren Auswirkungen auf das Funktionieren des Binnenmarkts, in: Amtsblatt der EU, Nr. L 193 vom 19. Juli 2016, S. 1-14. 
genen Ausgestaltung arbiträr und kann zu realen Verzerrungen führen. Die zudem anvisierte Einführung digitaler Betriebsstätten führt zu einer Ausweitung der Besteuerungsrechte auf reine Exportgeschäfte und kann das Risiko der Doppelbesteuerung erheblich erhöhen. In Anbetracht der Kritik an den Richtlinienvorschlägen und der vergleichsweise niedrigen Aufkommenseffekte ist die Umsetzung dieser Vorschläge nicht zu begrüßen. Die EU hat sich vorerst für eine Unterstützung der Vorhaben auf Ebene der OECD ausgesprochen. Der jetzige Trend der unilateralen Umsetzung der Digitalsteuer führt allerdings zu einer Zersplitterung im Binnenmarkt. Dies widerspricht dem eigentlichen Ziel eines stärker harmonisierten Ansatzes in der Fortentwicklung der europäischen Unternehmenssteuersysteme. Sollte es auf Ebene der OECD allerdings zu keiner Einigung über die Reformvorschläge kommen, werden die Richtlinienvorschläge der Europäischen Kommission voraussichtlich wieder auf die Tagesordnung gesetzt. Angesichts der in Steuerfragen notwendigen Einstimmigkeit kann allerdings aktuell nicht davon ausgegangen werden, dass die Umsetzung der Vorschläge in ihrer bisherigen Fassung höhere Erfolgschancen hätte. 